\title{
Spectral risk measure of holding stocks in the long run
}

\author{
Zsolt Bihary $^{1} \cdot$ Péter Csóka $^{1,2}$ (D) Dávid Zoltán Szabó ${ }^{3}$ \\ Published online: 18 June 2020 \\ (c) The Author(s) 2020
}

\begin{abstract}
We investigate how the spectral risk measure associated with holding stocks rather than a riskfree deposit, depends on the holding period. Previous papers have shown that within a limited class of spectral risk measures, and when the stock price follows specific processes, spectral risk becomes negative at long periods. We generalize this result for arbitrary exponential Lévy processes. We also prove the same behavior for all spectral risk measures (including the important special case of Expected Shortfall) when the stock price grows realistically fast and when it follows a geometric Brownian motion or a finite moment log stable process. This result would suggest that holding stocks for long periods has a vanishing downside risk. However, using realistic models, we find numerically that spectral risk initially increases for a significant amount of time and reaches zero level only after several decades. Therefore, we conclude that holding stocks has spectral risk for all practically relevant periods.
\end{abstract}

Keywords Coherent risk measures · Downside risk · Lévy processes · Finite moment log stable model $\cdot$ Time diversification

\section{JEL Classification G11}

\begin{abstract}
We would like to thank Peter Farkas, Gabor Kondor, Adam Zawadowski, and participants of the 8th Annual Financial Market Liquidity Conference for helpful comments. Péter Csóka was supported by the ÚNKP-17-4-III New National Excellence Program of the Ministry of Human Capacities and also thanks funding from National Research, Development and Innovation Office- NKFIH, K-120035.
\end{abstract}

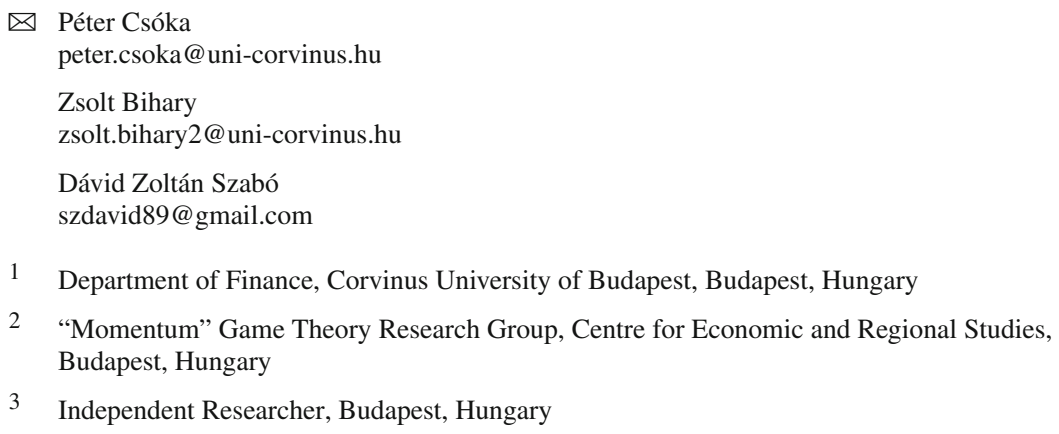




\section{Introduction}

There has been tremendous interest both from practitioners and researchers on the question of how risky it is to hold stocks rather than a risk-free deposit in the long run. The existence of time diversification, meaning that the risk of holding stocks decreases with the time horizon, has profound effects on all long-term investments such as pension savings and target date funds. Industry practitioners hold the common wisdom that the longer the intended holding period for a portfolio, the more it should contain risky, rather than risk-free investments.

The academic literature on the topic is more controversial (Bennyhoff 2009). Siegel (1998) showed that for investment horizons of at least 15 years, the risk of holding stocks (measured by realized variance) is lower than the risk of holding bonds or Treasury bills, and the risk of holding stocks is decreasing over time. However, Pástor and Stambaugh (2012) argue that stocks are more volatile in the long run from the perspective of an investor. The reason is that investors do not know the parameters of the stock price process (especially not the conditional expected return), and by making noisy predictions, at a 50-year horizon they face about 1.3 times higher return variance per year than at a 1-year horizon. Modeling the investors' beliefs, Avramov et al. (2017) find that there could be investors who perceive that the mean reversion of stocks is weaker, and hence they consider stocks riskier in the long run.

One can consider the probability that at time $t$ the stock investment value $S(t)$ is smaller than the risk free deposit value $S_{0} e^{r t}$, where $S_{0}$ is the initial investment and $r$ is a constant riskfree rate. This probability decreases with $t$, supporting the notion that the risk of the stock investment decreases with the holding period. However, as noted by Harlow (1991), this concept does not take into account the difference between $S_{0} e^{r t}$ and $S(t)$, and this difference can, of course, be larger and larger as $t$ increases.

Bodie (1995) approached the concept of risk differently by quantifying risk as the price of a put option that guarantees the risk-free payout $S_{0} e^{r t}$ at time $t$. The price of the corresponding put option was found to be increasing as $t$ increases, which can be interpreted as the risk of holding stocks increases over time. Wilkie (2001) criticized the methodology of Bodie (1995) by saying that if we wish to guarantee that we get at least the proceeds of a risk-free investment, then (since buying the option also has some costs) we have to invest everything in the risk-free investment, and pricing the option makes no sense. Moreover, Ferguson and Dean (1996) argued that the method of Bodie (1995) forces one to conclude that the risk of an asset relative to the risk of any other asset increases with the investment horizon, leading to a paradox.

Following Treussard (2006); Nguyen et al. (2012), we consider the risk of holding a stock rather than a risk-free deposit over time. More precisely, we analyze the spectral risk measure (Acerbi 2002) of the loss variable $Y(t)=S_{0} e^{r t}-S(t)$. If $Y(t)$ is positive (negative), then the stock performs poorly (well), in comparison with the risk free money market account. Spectral risk measures are coherent (Artzner et al. 1999, 2007) and can be expressed as a weighted average of losses, with increasing weights for higher losses. Thus we can think of the spectral risk of $Y(t)$ as a weighted average regret value associated with having bought the stock, rather than investing in a money market account.

As a special spectral risk, we consider Expected Shortfall (ES) (Acerbi and Tasche 2002; Rockafellar and Uryasev 2002), which can be computed and estimated efficiently (Acerbi and Tasche 2002) and is backtestable (Acerbi and Szekely 2014). Moreover, in 2013 the Basel Committee of Banking Supervision took the decision (Basel Committee 2013) of using ES 
for capital adequacy internal models, underscoring the importance of ES as a practical risk measure.

To describe the possible evolution of stock prices, we consider different stochastic processes. Besides the standard geometric Brownian motion (GBM) model, we analyze the exponential Lévy model (see for instance Mandelbrot (1963), Bertoin (1996), McCulloch (1996), Cai and Kou (2011), Fu and Yang (2012), Egami and Oryu (2015), and Fusai and Kyriakou (2016)). The exponential Lévy process, of which GBM is a special case, offers more flexibility to model the jumps and the heavy tail behavior of stock prices. As an illustration, we will investigate a particular exponential Lévy subclass called the Finite Moment Log Stable (FMLS) model (Carr and Wu 2003). In the FMLS model, moments of the logarithmic return exist on the gain side, but are infinite on the loss side. However, all moments exist for the price process, as the name FMLS indicates. Thus the FMLS price process can capture heavier tails for losses, while free from complications due to infinite moments.

There are two distinct regimes where the long-time behavior of risk is different. Depending on the parameters of the stock price process (the drift $\mu$ and the convexity adjustment $\kappa$ ), we distinguish between a high growth regime $(\mu>r+\kappa)$ and a medium growth regime $(r+\kappa \geq \mu>r)$. Our results extend those of Nguyen et al. (2012), since we treat the exponential Lévy model in general and the FMLS model in particular, and we have results for Expected Shortfall both in the GBM and in the FMLS models. We prove in case of a GBM stock process that in the high growth regime all spectral risk measures (including Expected Shortfall) will be negative at high $t$. We also investigate general exponential Lévy models and provide conditions under which in both at the high growth and at the medium growth regimes spectral risk will be negative at high $t$. For the FMLS model, we obtain the same results as for the GBM model, that is, in the high growth regime all spectral risk measures will be negative at high $t$.

This result would suggest that holding stocks for long periods has a vanishing downside risk. However, using realistic price process models, we find numerically that the spectral risk initially increases for a significant amount of time and reaches zero level only after several decades. This result indicates that holding stocks has spectral risk for all practically relevant periods. This conclusion is found to be insensitive to reasonable changes in parameters.

The structure of the paper is as follows. Spectral risk measures and their Choquet representation are given in Sect. 2. Section 3 contains the investigated stochastic processes, Sect. 4 provides the analytical results. Section 5 contains numerical results, Sect. 6 concludes.

\section{Spectral risk measures}

Consider the random variable $X$ that represents the loss distribution of a portfolio at the future time $t$, as seen at the current time. Thus positive values of $X$ are representing losses, negative values of $X$ are representing profits. The set of the random loss variables is denoted by $\mathcal{X}$.

Definition 2.1 The risk measure $\rho: \mathcal{X} \rightarrow \mathbb{R}$ is called a coherent risk measure (Artzner et al. $1999)$ if it satisfies the following properties:

- monotonicity: $Y \leq X \Rightarrow \rho(Y) \leq \rho(X)$,

- positive homogeneity: $h>0 \Rightarrow \rho(h X)=h \rho(X)$,

- sub-additivity: $\rho(X+Y) \leq \rho(X)+\rho(Y)$,

- translation invariance: $a \in \mathbb{R} \Rightarrow \rho(X+a)=\rho(X)+e^{-r t} a$,

where $e^{-r t}$ is the discount factor at time $t$. 
Throughout this paper we will assume a constant risk-free rate $r$. The discount factor $e^{-r t}$ is omitted in some papers, but it is especially important for us here, as we will investigate long horizons.

Coherent risk measures have a sound axiomatic foundation (Artzner et al. 1999), can be related to Bellman's principle, are supported by general equilibrium theory (Csóka et al. 2007) and they can also capture liquidity risk (Acerbi and Scandolo 2008). Moreover, coherent risk measures have also been used as an objective function in inventory problems (Ahmed et al. 2007; Philpott and De Matos 2012) considered the incorporation of a time-consistent coherent risk measure into a multi-stage stochastic programming model.

As a prominent coherent risk measure, Expected Shortfall (ES) (Acerbi and Tasche 2002; Rockafellar and Uryasev 2002) can be computed and estimated efficiently (Acerbi and Tasche 2002), and it is backtestable (Acerbi and Szekely 2014). Moreover, in 2013 the Basel Committee of Banking Supervision took the decision (Basel Committee 2013) of using ES for capital adequacy internal models. Given a random loss variable $X \in \mathcal{X}$ and a confidence level $\alpha \in[0,1], \mathrm{ES}_{\alpha}(X)$ is the average of the loss in the worst $100(1-\alpha) \%$ cases. As an example, $\alpha=90 \%$, and $\mathrm{ES}_{90 \%}$ is the average of the loss in the worst $10 \%$ of the cases.

To define ES formally in our setting, we need some more notation. Given a random loss variable $X \in \mathcal{X}$, let $F_{X}(x)=P[X \leq x]$ denote its distribution function. The generalized inverse of the loss distribution $X$ is defined as $F_{X}^{-1}(p)=\inf \left\{x \mid F_{X}(x) \geq p\right\}$.

Definition 2.2 Given a random loss variable $X \in \mathcal{X}$, for $\alpha \in[0,1)$, the Expected Shortfall of $X$ at $\alpha, \mathrm{ES}_{\alpha}(X)$ is defined as

$$
\operatorname{ES}_{\alpha}(X)=e^{-r t} \frac{1}{1-\alpha} \int_{\alpha}^{1} F_{X}^{-1}(p) \mathrm{d} p .
$$

For $\alpha=1, \mathrm{ES}_{1}$, that is the (discounted) maximum loss, is defined as

$$
\operatorname{ES}_{\alpha}(X)=e^{-r t} \text { ess. } \sup \{X\}
$$

where ess. $\sup \{X\}$ represents the essential supremum of the random loss variable $X$.

Spectral risk measures (Acerbi 2002) are also coherent and they are generalizing ES to get the weighted average of the losses with increasing weights as follows.

Definition 2.3 Given a random loss variable $X \in \mathcal{X}$, the spectral risk measure of $X, \rho_{\phi}(X)$ is defined as

$$
\rho_{\phi}(X)=e^{-r t} \int_{0}^{1} F_{X}^{-1}(p) \phi(p) \mathrm{d} p,
$$

where $\phi \in L^{1}([0,1])$, and the following properties are true:

- $\phi$ is positive;

- $\phi$ is monotonically increasing;

- $\int_{0}^{1}|\phi(p)| d p=1$.

Note again that all the usual formulas are written for losses, not gains, and they are adjusted with the $e^{-r t}$ discount factor. As it is clear from its definition, ES is a spectral risk measure that we will use as a specific example in our numerical calculations. Interestingly, Miller and Ruszczyński (2008) considered a portfolio optimization problem with different risk measures including spectral risk measures.

We will work with spectral risk measures throughout this paper, but we will use an equivalent definition derived as a special case of Choquet integrals (Choquet 1954). This formulation 
also appears in decision theory to model uncertainty (see for instance Grabisch and Labreuche (2010) and Labreuche and Grabisch (2018)). Choquet integral risk measures (see for instance Sriboonchita et al. (2009)) are defined as follows.

Definition 2.4 Given a random loss variable $X \in \mathcal{X}$, the Choquet integral risk measure of $X, \rho_{h}(X)$ is given by

$$
\rho_{h}(X)=e^{-r t} \int_{0}^{\infty} h\left(1-F_{X}(x)\right) \mathrm{d} x+e^{-r t} \int_{-\infty}^{0}\left[h\left(1-F_{X}(x)\right)-1\right] \mathrm{d} x,
$$

where $h$, the so-called distortion function (Wang 1996) is non-decreasing and satisfies $h(0)=$ 0 and $h(1)=1$.

Nguyen et al. (2012) notes that for concave distortion functions $h$ there exists a connection between Choquet integral risk measures and spectral risk measures such that if $h^{\prime}(1-p)=$ $\phi(p)$, then $\rho_{h}(X)=\rho_{\phi}(X)$ for all $X \in \mathcal{X}$. In this work, we will refer to spectral risk measures and Choquet integral risk measures interchangeably. A distortion function $h$ is non-degenerate, if there exists $p>0$ such that $h^{\prime}(p)>0$, or, equivalently, there exists $q<1$ such that $\phi(q)>0$.

Following Treussard (2006); Nguyen et al. (2012), we consider $Y(t)=S_{0} e^{r t}-S(t)$ as the loss variable whose spectral risk we want to calculate using Definition 2.4. If $Y(t)$ is positive (negative), then the stock performs poorly (well), in comparison with the risk-free deposit. We can also think of $Y(t)$ as a regret value associated with having bought the stock, rather than holding our investment in a risk-free deposit.

Throughout the paper, we will restrict our attention to non-degenerate distortion functions. This means that we preclude the degenerate maximum loss risk measure from further investigation.

\section{Investigated models}

Let $\{S(t)\}_{t \geq 0}$ be a positive valued stochastic process which represents the stock price on the market. As usual, this stochastic process is assumed to be defined on a complete filtered probability space $\left(\Omega, \mathcal{F},\left\{\mathcal{F}_{t}\right\}_{t \geq 0}, \mathbb{P}\right)$.

In the geometric Brownian motion model (GBM), the stock price follows the stochastic process

$$
S(t)=S_{0} e^{\left(\mu-\sigma^{2} / 2\right) t+\sigma W_{t}}, \quad S(0)=S_{0},
$$

where $\mu>0$ is the drift, $\sigma>0$ is the volatility, and $\left\{W_{t}\right\}_{t \geq 0}$ is a Wiener process.

The standardized log return of this process $\{S(t)\}_{t \geq 0}$ is distributed as

$$
\frac{\ln \left(\frac{S(t)}{S_{0}}\right)-\left(\mu-\sigma^{2} / 2\right) t}{\sigma \sqrt{t}} \sim \Phi[z],
$$

where $\Phi[z]$ is the standard normal distribution function.

Exponential Lévy processes, of which GBM is a special example, offer more flexibility to model heavier tail behaviour. In the exponential Lévy model, the stock price follows the stochastic process

$$
S(t)=S_{0} e^{\left(\mu-\kappa_{X}\right) t+X_{t}}, \quad S(0)=S_{0}
$$


where $X_{t}$ is a Lévy process fully determined by its Lévy triplet $\mathbb{T}, \mu$ is the drift, and $\kappa_{X}$ is the convexity adjustment term that can be calculated as

$$
\kappa_{X}=\frac{1}{t} \ln \mathbb{E} e^{X_{t}} .
$$

The convexity adjustment $\kappa_{X}$ plays the role of an exponential compensator in the sense that the process $\left\{e^{-\kappa_{X} t+X_{t}}\right\}_{t \geq 0}$ is an $\left(\left\{\mathcal{F}_{t}\right\}_{t \geq 0}, \mathbb{P}\right)$-exponential-martingale. Note that $\kappa_{X}$ does not depend on time. We will denote the distribution function of the Lévy process at a given time $t$ by $F_{t}$.

We will consider a specific exponential Lévy model, called Finite Moment Log Stable (FMLS) model. In the FMLS model, the stock price movement follows the stochastic process (Carr and $\mathrm{Wu}$ 2003)

$$
S(t)=S_{0} e^{(\mu-\kappa) t+\sigma L_{t}^{\alpha}}, \quad S(0)=S_{0},
$$

where $\mu$ is the drift, $\sigma$ is the scale of volatility, $L_{t}^{\alpha}$ is alpha stable distributed with shape parameters $\alpha \in(1,2)$ and $\beta=-1$, with scale parameter $t^{\frac{1}{\alpha}}$, and with zero mean, that is $L_{t}^{\alpha} \sim S\left(\alpha,-1, t^{\frac{1}{\alpha}}, 0\right)$ (see more details in Carr and $\mathrm{Wu}(2003)$ ). Note that the only remaining free parameter for the distribution is $\alpha$. The $\alpha \rightarrow 2$ limit corresponds to the GBM model. Decreasing $\alpha$ results in heavier tails for the losses. As before, $\kappa$ is the convexity adjustment term for the FMLS model, given as

$$
\kappa=-\sigma^{\alpha} \sec (\pi \alpha / 2) .
$$

Note that $\kappa$ is positive due to the properties of the $\sec$ function, as $\alpha \in(1,2)$. Now the $\log$ return of the $S(t)$ process becomes

$$
\ln \left(\frac{S(t)}{S_{0}}\right)=(\mu-\kappa) t+\sigma L_{t}^{\alpha},
$$

therefore its distribution is $S\left(\alpha,-1, \sigma t^{\frac{1}{\alpha}},(\mu-\kappa) t\right)$ for a fixed $t$. Stable distributions have the following important property (see for example page 24 of Janicki and Weron (1994)).

Proposition 3.1 If $X$ is an alpha stable distributed random variable $X \sim S(\alpha, \beta, 1,0)$ then $\gamma X+\delta \sim S(\alpha, \beta, \gamma, \delta)$ for $\delta>0$ and $\gamma \in \mathbb{R}$.

Using Proposition 3.1 we obtain

$$
\frac{\ln \left(\frac{S(t)}{S_{0}}\right)-(\mu-\kappa) t}{\sigma t^{\frac{1}{\alpha}}} \sim S(\alpha,-1,1,0) .
$$

We denote the distribution and density functions of $S(\alpha,-1,1,0)$ at $z$ by $\Theta_{\alpha}(z)$ and $\Theta_{\alpha}^{\prime}(z)$, respectively.

\section{Analytical results}

The main question of this paper is the long horizon behavior of the risk curve $\rho_{h}(Y(t))$. To give some insights, let us discuss some general qualitative features of the risk curve. At $t=0$, $\rho_{h}(Y(0))=0$, since initially the stock price is deterministic. Roughly speaking, there are two quantities affecting spectral risk, the mean and dispersion of the stock price distribution. Greater mean leads to lower risk, while greater dispersion results in higher risk. For short times, the dispersion grows much faster than the mean, leading to initially increasing risk 
curves. The question is whether risk starts to decrease at a certain time or keeps rising. If $\rho_{h}(Y(t))$ becomes negative for a large $t$, it must have decreased from its positive maximum. The above described qualitative features of the risk curve will be demonstrated with numerical examples in Sect. 5.

As discussed in the literature (see Hull (2012) and Ross (2015)), we will work under the statistical (real-world) measure in this paper. There are two distinct regimes where the long-time behavior of risk is different. The case when $\mu>r+\kappa$ we call the high growth regime. When $r+\kappa \geq \mu>r$, we are in the medium growth regime. The case $\mu \leq r$ (low growth regime) is not realistic, as it would mean that the risky asset grows slower on average than the risk-free asset.

Nguyen et al. (2012) investigated two exponential Lévy Processes, GBM and exponential Poisson. They proved that $h^{\prime}>c>0$ is a sufficient condition in both regimes for the risk to be negative at high $t$. In the high growth regime, they also proved this behavior if $h^{\prime}$ is continuous. We reinvestigated the GBM model and proved the new result that in the high growth regime all spectral risk measures would be negative at high $t$. Moreover, for general exponential Lévy models, we proved that in both high growth and medium growth regimes if $h^{\prime}>c>0$, then spectral risk will be negative at high $t$. We also investigated a specific exponential Lévy model, the FMLS model, and we obtained the same results as for the GBM model, that is in the high growth regime all spectral risk measures will be negative at high $t$.

\subsection{GBM model}

In this section, we assume that the stock process $S(t)$ is a geometric Brownian motion.

The formula derived by Nguyen et al. (2012) for the spectral risk, adjusted to our definition that takes into account discounting, is:

$$
\rho_{h}(Y(t))=S_{0}\left[1-e^{(\mu-r) t} \int_{-\infty}^{\infty} h^{\prime}(\Phi[z]) \frac{1}{\sqrt{2 \pi}} e^{\frac{-1}{2}(z-\sigma \sqrt{t})^{2}} \mathrm{~d} z\right] .
$$

We have the following own result.

Theorem 4.1 In the GBM stock price model, $\rho_{h}(Y(t))$ is negative for a large $t$ if $\mu>r+\frac{\sigma^{2}}{2}$.

Proof Since $h^{\prime}$ is a monotonically decreasing function in a closed interval, it can only have a countable number of jump points, so the function is almost surely continuous everywhere.

Let $A=[0, d]$. Since we only consider non-degenerate distortion functions,

$$
h^{\prime}(x) \geq c \quad \forall \quad x \in A,
$$

where $c>0$. For the integral in (9), we obtain the following lower bound.

$$
\begin{aligned}
& \int_{-\infty}^{\infty} h^{\prime}(\Phi[z]) \frac{1}{\sqrt{2 \pi}} e^{\frac{-1}{2}(z-\sigma \sqrt{t})^{2} \mathrm{~d} z} \\
& \quad=\int_{\Phi^{-1} A} h^{\prime}(\Phi[z]) \frac{1}{\sqrt{2 \pi}} e^{\frac{-1}{2}(z-\sigma \sqrt{t})^{2}} \mathrm{~d} z+\int_{R \backslash \Phi^{-1} A} h^{\prime}(\Phi[z]) \frac{1}{\sqrt{2 \pi}} e^{\frac{-1}{2}(z-\sigma \sqrt{t})^{2}} \mathrm{~d} z \\
& \quad \geq c \int_{\Phi^{-1} A} \frac{1}{\sqrt{2 \pi}} e^{\frac{-1}{2}(z-\sigma \sqrt{t})^{2}} \mathrm{~d} z+\int_{R \backslash \Phi^{-1} A} h^{\prime}(\Phi[z]) \frac{1}{\sqrt{2 \pi}} e^{\frac{-1}{2}(z-\sigma \sqrt{t})^{2}} \mathrm{~d} z .
\end{aligned}
$$

By definition, $\Phi^{-1}(A)=(-\infty, b]$, where $b=\Phi^{-1}(d)$. For every point $x$ on the interval $(-\infty, b]$ we have $h^{\prime}(\Phi[x]) \geq c$. Let us choose a subinterval $\left[a^{\prime}, b\right]$ of the interval $(-\infty, b]$. 
Since $h^{\prime} \geq 0$ everywhere, we can assess that

$$
\begin{aligned}
& \int_{-\infty}^{\infty} h^{\prime}(\Phi[z]) \frac{1}{\sqrt{2 \pi}} e^{\frac{-1}{2}(z-\sigma \sqrt{t})^{2}} \mathrm{~d} z \\
& \quad \geq c \int_{a^{\prime}}^{b} \frac{1}{\sqrt{2 \pi}} e^{\frac{-1}{2}(z-\sigma \sqrt{t})^{2}} \mathrm{~d} z=c\left[\Phi(b-\sigma \sqrt{t})-\Phi\left(a^{\prime}-\sigma \sqrt{t}\right)\right],
\end{aligned}
$$

where the last equation follows from integrating the density function of a normally distributed random variable with mean $\sigma \sqrt{t}$ and standard deviation 1 on the interval $\left[a^{\prime}, b\right]$.

Using the mean value theorem, for a $d \in\left[a^{\prime}, b\right]$ we assess that

$$
c\left[\Phi(b-\sigma \sqrt{t})-\Phi\left(a^{\prime}-\sigma \sqrt{t}\right)\right] \geq c\left(b-a^{\prime}\right) e^{\frac{-1}{2}(d-\sigma \sqrt{t})^{2}} .
$$

Therefore, we can deduce that

$$
\begin{aligned}
& e^{(\mu-r) t} \int_{-\infty}^{\infty} h^{\prime}(\Phi[z]) \frac{1}{\sqrt{2 \pi}} e^{\frac{-1}{2}(z-\sigma \sqrt{t})^{2}} \mathrm{~d} z \\
& \quad \geq e^{(\mu-r) t} c \cdot\left(b-a^{\prime}\right) e^{\frac{-1}{2}(d-\sigma \sqrt{t})^{2}}=c \cdot\left(b-a^{\prime}\right) e^{\left(\mu-r-\frac{\sigma^{2}}{2}\right) t+d \sigma \sqrt{t}-\frac{1}{2} d^{2}} .
\end{aligned}
$$

The last expression goes to infinity if $t$ goes to infinity, since we assumed that $\mu-r-\frac{\sigma^{2}}{2}>0$. It follows that $\rho_{h}(Y(t))$ in (9) goes to minus infinity and hence becomes negative for a large $t$.

\subsection{Arbitrary exponential Lévy models}

In this section, we assume that the stock process $S(t)$ is an exponential Lévy process. For a brief introduction, see Sect. 3. First, we derive an own formula for the spectral risk in this model.

Theorem 4.2 In an arbitrary exponential Lévy model, the spectral risk of $Y(t)$ is

$$
\rho_{h}(Y(t))=S_{0}\left[1-e^{(\mu-r) t} \int_{-\infty}^{\infty} e^{z-\kappa_{X}(1) t} h^{\prime}\left(F_{t}(z)\right) f_{t}(z) \mathrm{d} z\right],
$$

where $F_{t}$ is the distribution function of the Lévy process at a given time $t$, and $f_{t}$ is its density function.

Proof Using the Choquet representation in Definition 2.4, we get that

$$
\rho_{h}(Y(t))=\int_{0}^{\infty} h\left(1-F_{Y(t)}(x)\right) d x+\int_{-\infty}^{0}\left[h\left(1-F_{Y(t)}(x)\right)-1\right] \mathrm{d} x .
$$

We conduct a substitution to express this formula with the distribution function of the Lévy process. To do so, we further shape $1-F_{Y(t)}(x)$. We know that the log return of the stock in this model can be written as

$$
\ln \left(\frac{S(t)}{S_{0}}\right)=\mu t+X_{t}-t \kappa_{X}
$$


Using Eq. (16), we obtain the following

$$
\begin{aligned}
1 & -F_{Y(t)}(x)=\mathbb{P}\left(S(t)<S_{0} e^{r t}-x\right) \\
& =\mathbb{P}\left(X(t)<\ln \left(\frac{S_{0} e^{r t}-x}{S_{0}}\right)-\left(\mu-\kappa_{X}(1)\right) t\right) \\
& =F_{t}\left(\ln \left(\frac{S_{0} e^{r t}-x}{S_{0}}\right)-\left(\mu-\kappa_{X}(1)\right) t\right) .
\end{aligned}
$$

In Eq. (15), performing the substitution $z=\ln \left(e^{r t}-\frac{x}{S_{0}}\right)-\left(\mu-\kappa_{X}\right) t \Leftrightarrow d x=$ $-S_{0} e^{z+\left(\mu-\kappa_{X}\right) t} d z$, we get that

$$
\rho_{h}(Y(t))=\int_{-\infty}^{C} h\left(F_{t}(z)\right) S_{0} e^{z+\left(\mu-\kappa_{X}\right) t} \mathrm{~d} z+\int_{C}^{\infty}\left[h\left(F_{t}(z)\right)-1\right] S_{0} e^{z+\left(\mu-\kappa_{X}\right) t} \mathrm{~d} z,
$$

where $C=\left(r-\mu+\kappa_{X}\right) t$. Integrating by parts, we obtain that

$$
\begin{aligned}
& \rho_{h}(Y(t))=\left[h\left(F_{t}(z)\right) S_{0} e^{z+\left(\mu-\kappa_{X}\right) t}\right]_{-\infty}^{C}-\int_{-\infty}^{C} h^{\prime}\left(F_{t}(z)\right) f_{t}(z) S_{0} e^{z+\left(\mu-\kappa_{X}\right) t} \mathrm{~d} z \\
& +\left[h\left(F_{t}(z)-1\right] S_{0} e^{z+\left(\mu-\kappa_{X}\right) t}\right]_{C}^{\infty}-\int_{C}^{\infty} h^{\prime}\left(F_{t}(z)\right) f_{t}(z) S_{0} e^{z+\left(\mu-\kappa_{X}\right) t} \mathrm{~d} z .
\end{aligned}
$$

Substituting the values on the boundary points using $h(0)=0, h(1)=1$ we can assert that

$$
\rho_{h}(Y(t))=S_{0} e^{r t}\left[1-e^{(\mu-r) t} \int_{-\infty}^{\infty} e^{z-\kappa_{X} t} h^{\prime}\left(F_{t}(z)\right) f_{t}(z) \mathrm{d} z\right]
$$

Applying Theorem 4.2, we prove the same long-time behavior for arbitrary Lévy models as was proven by Nguyen et al. (2012) in the case of GBM.

Theorem 4.3 In an arbitrary exponential Lévy model $\rho_{h}(Y(t))$ is negative for a large $t$ if $h^{\prime}(\cdot)$ is bounded below with a $c>0$ constant, and if $\mu>r$.

Proof Due to the definition of $\kappa_{X}$ and by the assumption of the theorem

$$
\begin{aligned}
& \int_{-\infty}^{\infty} e^{z-\kappa_{X} t} h^{\prime}\left(F_{t}(z)\right) f_{t}(z) \mathrm{d} z \\
& \quad \geq C \cdot \int_{-\infty}^{\infty} e^{z-\kappa_{X} t} f_{t}(z) \mathrm{d} z=C \cdot e^{-\kappa_{X} t} \mathbb{E}\left[e^{X_{t}}\right]=C .
\end{aligned}
$$

and therefore,

$$
e^{(\mu-r) t} \int_{-\infty}^{\infty} e^{z-\kappa_{X}(1) t} h^{\prime}\left(F_{t}(z)\right) f_{t}(z) \mathrm{d} z
$$

goes to infinity as $t$ goes to infinity, implying that $\rho_{h}(Y(t))$ also goes to minus infinity and $\rho_{h}(Y(t))$ becomes negative for a large $t$.

\subsection{FMLS model}

In this section, we assume that the stock process $S(t)$ is an FMLS process. For a brief introduction, see Sect. 3. 
Theorem 4.4 In the FMLS stock price model, the spectral risk of $Y(t)$ is

$$
\rho_{h}(Y(t))=S_{0}\left[1-e^{(\mu-r) t} \int_{-\infty}^{\infty} e^{z \sigma t^{1 / \alpha}-\kappa t} h^{\prime}\left(\Theta_{\alpha}(z)\right) \Theta_{\alpha}^{\prime}(z) \mathrm{d} z\right] .
$$

Proof The proof is similar to that of Theorem 4.2. The main difference appears in the variable substitution. Due to the properties of the stable variables (Proposition 3.1) we can express the risk without time dependency.

Performing the substitution $z=\frac{\ln \left(e^{r t}-\frac{x}{S_{0}}\right)-(\mu-\kappa) t}{\sigma t^{\frac{1}{\alpha}}} \Leftrightarrow d x=-S_{0} \sigma t^{\frac{1}{\alpha}} e^{z \sigma t^{\frac{1}{\alpha}}+(\mu-\kappa) t} d z$, integrating by parts and utilising $h(0)=0$ and $h(1)=1$, we obtain $\rho_{h}(Y(t))$ as stated in the theorem.

Theorem 4.5 In the FMLS stock price model, $\rho_{h}(Y(t))$ is negative for a large $t$ if $h^{\prime}(\cdot)$ is bounded below with a $c>0$ constant, and if $\mu>r$.

Proof This is a special case of Theorem 4.3, since the FMLS process is an exponential Lévy process.

Theorem 4.6 In the FMLS stock price model, $\rho_{h}(Y(t))$ is negative for a large $t$ if $\mu>r+\kappa$.

Proof Since $h^{\prime}$ is a monotonically decreasing function in a closed interval, it can only have a countable number of jump points, so the function is almost surely continuous everywhere.

Let $A=[0, d]$. Since we only consider non-degenerate distortion functions,

$$
h^{\prime}(x) \geq c \quad \forall \quad x \in A,
$$

where $c>0$. As for the integral in (22), we obtain the following lower bound.

$$
\begin{aligned}
& \int_{-\infty}^{\infty} e^{z \sigma t^{1 / \alpha}-\kappa t} h^{\prime}\left(\Theta_{\alpha}(z)\right) \Theta_{\alpha}^{\prime}(z) \mathrm{d} z \\
& \quad=\int_{\Theta_{\alpha}^{-1}(A)} e^{z \sigma t^{1 / \alpha}-\kappa t} h^{\prime}\left(\Theta_{\alpha}(z)\right) \Theta_{\alpha}^{\prime}(z) \mathrm{d} z+\int_{R \backslash \Theta_{\alpha}^{-1}(A)} e^{z \sigma t^{1 / \alpha}-\kappa t} h^{\prime}\left(\Theta_{\alpha}(z)\right) \Theta_{\alpha}^{\prime}(z) \mathrm{d} z \\
& \quad \geq c \int_{\Theta_{\alpha}^{-1}(A)} e^{z \sigma t^{1 / \alpha}-\kappa t} \Theta_{\alpha}^{\prime}(z) \mathrm{d} z+\int_{R \backslash \Theta_{\alpha}^{-1}(A)} e^{z \sigma t^{1 / \alpha}-\kappa t} h^{\prime}\left(\Theta_{\alpha}(z)\right) \Theta_{\alpha}^{\prime}(z) \mathrm{d} z
\end{aligned}
$$

By definition, $\Theta_{\alpha}^{-1}(A)=(-\infty, b]$, where $b=\Theta_{\alpha}^{-1}(a)$. For every point $x$ on the interval $(-\infty, b]$, it holds that $h^{\prime}\left(\Theta_{\alpha}(x)\right) \geq c$.

Next, we will show that $\Theta_{\alpha}^{\prime}(z)$ is positive on an appropriate subinterval $\left[a^{\prime}, b^{\prime}\right]$ of $[-\infty, b]$. We know that $\Theta_{\alpha}^{\prime}(z)$ is the density function of a stable distributed random variable with parameters $(\alpha,-1,1,0)$. Moreover, on $[-\infty, b]$ the cumulative distribution function of this random variable is not constant, hence the density function is surely positive at some points. According to Proposition 3.12 on page 102 of Cont and Tankov (2004), if a Lévy process is infinitely active, then the density function of this process is continuous for every $t$. Carr and $\mathrm{Wu}$ (2003) showed that the stable process is infinitely active in case $1<\alpha<2$, meaning that it has an infinite number of jumps over any time interval. Hence we can assess that the process $L_{t}^{\alpha,-1}$ has continuous density function for every $t \geq 0$. In particular, it holds for $t=1$, that is for $\Theta_{\alpha}^{\prime}(z)$. Thus $\Theta_{\alpha}^{\prime}(z)$ is positive in at least one point on $[-\infty, b]$, implying that $\Theta_{\alpha}^{\prime}(z)$ is positive on an appropriate subinterval $\left[a^{\prime}, b^{\prime}\right]$ of $[-\infty, b]$. 
Denote the minimum value of $\Theta_{\alpha}^{\prime}(z)$ on $\left[a^{\prime}, b^{\prime}\right]$ by $c^{\prime}>0$. Since $h^{\prime} \geq 0$ everywhere, we obtain that

$$
\begin{aligned}
& e^{(\mu-r) t} \int_{-\infty}^{\infty} e^{z \sigma t^{1 / \alpha}-\kappa t} h^{\prime}\left(\Theta_{\alpha}(z)\right) \Theta_{\alpha}^{\prime}(z) \mathrm{d} z \\
& \quad \geq c \cdot e^{(\mu-r) t} \int_{a^{\prime}}^{b^{\prime}} e^{z \sigma t^{1 / \alpha}-\kappa t} \Theta_{\alpha}^{\prime}(z) \mathrm{d} z=\int_{a^{\prime}}^{b^{\prime}} c \cdot e^{(\mu-r-\kappa) t+z \sigma t^{1 / \alpha}} \Theta_{\alpha}^{\prime}(z) \mathrm{d} z .
\end{aligned}
$$

Since $\Theta_{\alpha}^{\prime}(z)$ is positive on an appropriate subinterval $\left[a^{\prime}, b^{\prime}\right]$ of $[-\infty, b]$, we obtain that

$$
\begin{gathered}
e^{(\mu-r) t} \int_{-\infty}^{\infty} e^{z \sigma t^{1 / \alpha}-\kappa t} h^{\prime}\left(\Theta_{\alpha}(z)\right) \Theta_{\alpha}^{\prime}(z) \mathrm{d} z \\
\geq c \cdot c^{\prime} \int_{a^{\prime}}^{b^{\prime}} e^{(\mu-r-\kappa) t+z \sigma t^{1 / \alpha}} \mathrm{d} z .
\end{gathered}
$$

By integrating, it follows that

$$
\begin{aligned}
& \int_{a^{\prime}}^{b^{\prime}} e^{(\mu-r-\kappa) t+z \sigma t^{1 / \alpha}} \mathrm{d} z=\left[\frac{e^{(\mu-r-\kappa) t+z \sigma t^{1 / \alpha}}}{\sigma t^{1 / \alpha}}\right]_{a^{\prime}}^{b^{\prime}} \\
& =\frac{e^{(\mu-r-\kappa) t}}{\sigma t^{1 / \alpha}}\left[e^{b^{\prime} \sigma t^{1 / \alpha}-a^{\prime} \sigma t^{1 / \alpha}}\right]=\frac{e^{(\mu-r-\kappa) t}}{\sigma t^{1 / \alpha}}\left[e^{\left(b^{\prime}-a^{\prime}\right) \sigma t^{1 / \alpha}}\right] .
\end{aligned}
$$

The last expression goes to infinity if $t$ goes to infinity, since we assumed that $\mu>r+\kappa$. It follows that $\rho_{h}(Y(t))$ in (22) goes to minus infinity and hence becomes negative for a large $t$.

\section{Numerical results}

To illustrate the implications of our theoretical calculations, in this section we present numerical results performed with realistic model parameters. We obtained the daily closing prices on the dividend-adjusted SP500 index to represent the risky "stock" in our model. The cash deposit rate was estimated using data on the annualized returns on 3-month US treasury bills. Both time series were taken from 1960 to 2016. Calibrating the historical data to the GBM model yielded the following estimation for the parameters: $r=0.048, \mu=0.089$, $\sigma_{G B M}=0.155$.

To illustrate the effect of stronger tails in the loss distribution, and to offer some robustness test, we also calibrated a set of FMLS models with four different $\alpha$ parameters: $\alpha=1.8,1.85,1.9,1.95$. We used the same $r=0.048, \mu=0.089$ values as for the GBM model. At each $\alpha$, we set $\sigma_{\alpha}$ such that the difference between the first and the third yearly return quartiles are matched with that of the GBM model. This procedure ensures consistency between the different models in terms of short-term volatility. All five of these models represent realistic test cases for long-term investments in a well-diversified US equity portfolio.

All the models are in the "high growth regime" as defined in Sect. 4. Using our results from Sect. 4, we computed the Expected Shortfall at the $90 \%$ level as an illustrative risk measure. We performed the calculation for all five calibrated models for different holding periods, up to one hundred years, with monthly steps. The value of the initial stock investment was set to unity. The distribution functions of the normal and stable distributions are readily available in MatLab, the necessary integrations were performed with numerical quadrature, also available in MatLab. The results are plotted in Fig. 1. The risk curves start at zero, 
increase up to a maximum value, then, in accordance with our analytical results, drop down to and below zero. At any given holding period, the GBM model produces the lowest risk levels, while the FMLS curves, especially the ones with relatively low $\alpha$ parameters, show higher risk values. This is due to the heavier tails in the FMLS distribution on the loss side.

As discussed in the seminal paper of Artzner et al. (1999), coherent risk measures can be interpreted as the amount of reserve cash necessary to lower market exposure to a tolerable level. Although the numerical results shown underscore our analytic conclusion that the risk measure becomes zero at some holding time for all the investigated models, note the time scale on the $x$ axis. The necessary reserve cash becomes zero only at around, or beyond, one hundred years. The risk of holding stocks remains significant even after one hundred years, when the stock return distribution has heavy tails (see the curves with the smallest alpha values).

On the other hand, at long but realistic holding periods (a few decades), we need quite a sizable cushion to mitigate risk. According to Fig. 1, the necessary cash reserve amounts to about half of the initial stock value.

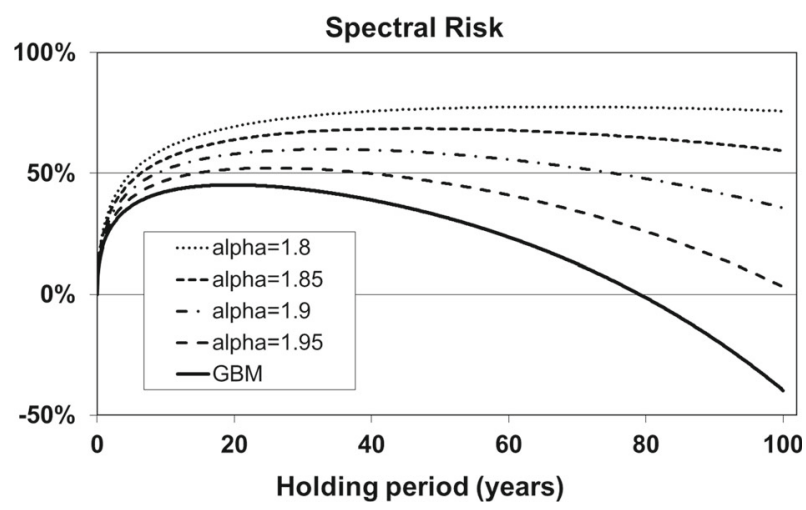

Fig. 1 Expected Shortfall (90\%) risk measure for holding a stock rather than a risk-free deposit, as a function of the holding period for $r=0.048, \mu=0.089$, and $\sigma_{G B M}=0.155$. Risk curves are plotted for the GBM model, and for the FMLS model with different $\alpha$ parameters

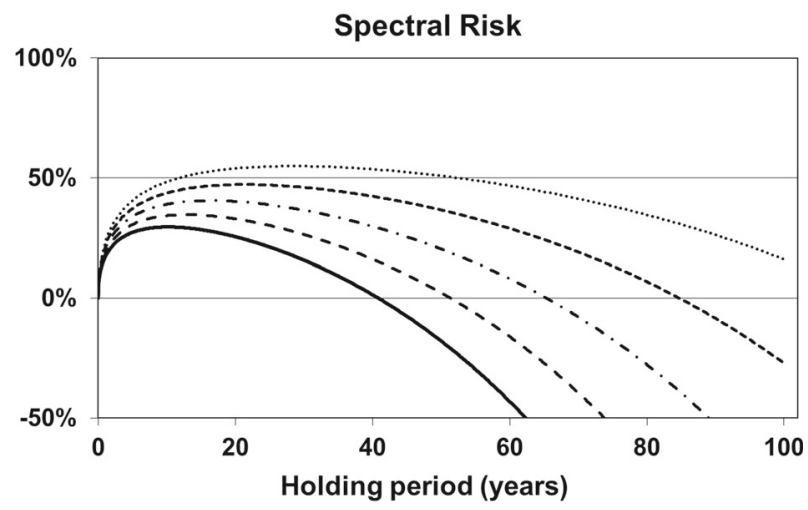

Fig. 2 Expected Shortfall (90\%) risk measure for holding a stock rather than a risk-free deposit, as a function of the holding period for $r=0.048, \mu=0.089$, and $\sigma_{G B M}=0.125$. Risk curves are plotted for the GBM model, and for the FMLS model with different $\alpha$ parameters 


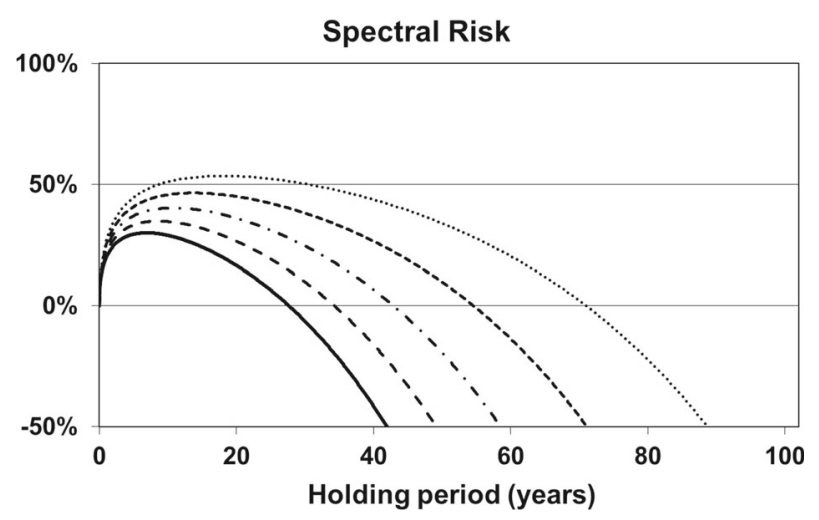

Fig. 3 Expected Shortfall (90\%) risk measure for holding a stock rather than a risk-free deposit, as a function of the holding period for $r=0.048, \mu=0.11$, and $\sigma_{G B M}=0.155$. Risk curves are plotted for the GBM model, and for the FMLS model with different $\alpha$ parameters

To perform some sensitivity tests, let us consider the effect of changing the parameters. In particular, we will explore parameter modifications that lower the risk measure. For most users, decreasing the $90 \%$ confidence level for the Expected Shortfall is not an option. Increasing the confidence level would lead to higher risks, since then the average of even worse outcomes would be calculated. To decrease risk, volatility should be decreased or the difference between $\mu$ and $r$ should be increased in our models. In Fig. 2, we show risk curves with a reduced volatility of $\sigma_{G B M}=0.125$. In Fig. 3, the curves are calculated with the increased drift of $\mu=0.11$. All other parameters are the same as in Fig. 1. Our models with these modified parameters represent optimistic, but still realistic market views. Although the risk curves with the modified parameters shifted down as expected, our conclusions remain essentially the same: Risk remains significant for decades.

\section{Conclusion}

For long-run investors, it is crucial to know how to evaluate the risk associated with holding stocks rather than a risk-free deposit. We analyzed spectral risk, which can be interpreted as a weighted average regret value associated with having bought the stock, rather than investing in a money market account.

We show that for arbitrary exponential Lévy processes and when all possible outcomes have strictly positive weight, spectral risk becomes negative at long periods. We also prove the same behavior for all spectral risk measures (including the important special case of Expected Shortfall) when the stock price is in the high-growth regime and when it follows a GBM or an FMLS process.

We leave it as an open question whether Expected Shortfall becomes negative for arbitrary exponential Lévy processes in the high growth regime. Moreover, in a more realistic model, as well as in standard industry practice, for such long time horizons a stochastic interest rate model would be necessary. Since coherent measures of risk are extended to a dynamic setting with stochastic interest rates by Riedel (2004), it would be interesting to consider a model with a correlated joint distribution of the future stock price and the discount factor. 
Our results show that in the long run, the steady growth of the expected stock price overrides its uncertainty in terms of the risk involved. However, how long is the long run in practice? To answer this question, we calibrated the models to historical market data and performed numerical calculations. We find that the risk as defined in our paper indeed becomes negative, but it remains significantly positive for decades, even for optimistic market views. Increasing the tails in the FMLS process, or adding model or parameter uncertainty makes the situation even worse. Also supported by the sensitivity tests, our final conclusion is that holding stocks is risky for all relevant holding periods.

Acknowledgements Open access funding provided by Corvinus University of Budapest (BCE).

Open Access This article is licensed under a Creative Commons Attribution 4.0 International License, which permits use, sharing, adaptation, distribution and reproduction in any medium or format, as long as you give appropriate credit to the original author(s) and the source, provide a link to the Creative Commons licence, and indicate if changes were made. The images or other third party material in this article are included in the article's Creative Commons licence, unless indicated otherwise in a credit line to the material. If material is not included in the article's Creative Commons licence and your intended use is not permitted by statutory regulation or exceeds the permitted use, you will need to obtain permission directly from the copyright holder. To view a copy of this licence, visit http://creativecommons.org/licenses/by/4.0/.

\section{References}

Acerbi, C. (2002). Spectral measures of risk: A coherent representation of subjective risk aversion. Journal of Banking \& Finance, 26, 1505-1518.

Acerbi, C., \& Scandolo, G. (2008). Liquidity risk theory and coherent measures of risk. Quantitative Finance, $8(7), 681-692$.

Acerbi, C., \& Szekely, B. (2014). Back-testing expected shortfall. Risk, 27(11), 76-81.

Acerbi, C., \& Tasche, D. (2002). Expected shortfall: A natural coherent alternative to value at risk. Economic Notes, 31, 379-388.

Ahmed, S., Çakmak, U., \& Shapiro, A. (2007). Coherent risk measures in inventory problems. European Journal of Operational Research, 182(1), 226-238.

Artzner, P., Delbaen, F., Eber, J. M., \& Heath, D. (1999). Coherent measures of risk. Mathematical Finance, 9, 203-228.

Artzner, P., Delbaen, F., Eber, J.-M., Heath, D., \& Ku, H. (2007). Coherent multiperiod risk adjusted values and Bellman's principle. Annals of Operations Research, 152(1), 5-22.

Avramov, D., Cederburg, S., \& Lučivjanská, K. (2017). Are stocks riskier over the long run? Taking cues from economic theory. The Review of Financial Studies, 31(2), 556-594.

Basel Committee. (2013). Fundamental review of the trading book: A revised market risk framework. Consultative Document, October.

Bennyhoff, D. G. (2009). Time diversification and horizon-based asset allocations. The Journal of Investing, $18(1), 45-52$.

Bertoin, J. (1996). Lévy processes. Cambridge: Cambridge University Press.

Bodie, Z. (1995). On the risk of stocks in the long run. Financial Analysts Journal, 51, 18-22.

Cai, N., \& Kou, S. G. (2011). Option pricing under a mixed-exponential jump diffusion model. Management Science, 57(11), 2067-2081.

Carr, P., \& Wu, L. (2003). The finite moment log stable process and option pricing. The Journal of Finance, $8(2), 753-778$.

Choquet, G. (1954). Theory of capacities. Annales de l'institut Fourier, 5, 131-295.

Cont, R., \& Tankov, P. (2004). Financial modelling with jump processes. New York: Chapman and Hall/CRC.

Csóka, P., Herings, P. J.-J., \& Kóczy, L. Á. (2007). Coherent measures of risk from a general equilibrium perspective. Journal of Banking \& Finance, 31(8), 2517-2534.

Egami, M., \& Oryu, T. (2015). An excursion-theoretic approach to regulator's bank reorganization problem. Operations Research, 63(3), 527-539.

Ferguson, R., \& Dean, L. (1996). On the risk of stocks in the long run: A Comment. Financial Analysts Journal, 52, 67-68. 
Fu, J., \& Yang, H. (2012). Equilibruim approach of asset pricing under Lévy process. European Journal of Operational Research, 223(3), 701-708.

Fusai, G., \& Kyriakou, I. (2016). General optimized lower and upper bounds for discrete and continuous arithmetic Asian options. Mathematics of Operations Research, 41(2), 531-559.

Grabisch, M., \& Labreuche, C. (2010). A decade of application of the Choquet and Sugeno integrals in multi-criteria decision aid. Annals of Operations Research, 175(1), 247-286.

Harlow, W. V. (1991). Asset allocation in a downside-risk framework. Financial Analysts Journal, 47(5), $28-40$.

Hull, J. C. (2012). Risk management and financial institutions (Vol. 806). Hoboken: Wiley.

Janicki, A., \& Weron, A. (1994). Simulation and Chaotic behavior of alpha-stable stochastic processes. New York: Marcel Dekker.

Labreuche, C., \& Grabisch, M. (2018). Using multiple reference levels in multi-criteria decision aid: The generalized-additive independence model and the Choquet integral approaches. European Journal of Operational Research, 267(2), 598-611.

Mandelbrot, B. (1963). New methods in statistical economics. Journal of Political Economy, 71, 421-440.

McCulloch, J. H. (1996). Financial applications of stable distributions. Handbook of Statistics, 14, 393-425.

Miller, N., \& Ruszczyński, A. (2008). Risk-adjusted probability measures in portfolio optimization with coherent measures of risk. European Journal of Operational Research, 191(1), 193-206.

Nguyen, H. T., Pham, U. H., \& Tran, H. D. (2012). On some claims related to Choquet integral risk measures. Annals of Operations Research, 195, 5-31.

Pástor, L., \& Stambaugh, R. F. (2012). Are stocks really less volatile in the long run? The Journal of Finance, 67(2), 431-478.

Philpott, A. B., \& De Matos, V. L. (2012). Dynamic sampling algorithms for multi-stage stochastic programs with risk aversion. European Journal of Operational Research, 218(2), 470-483.

Riedel, F. (2004). Dynamic coherent risk measures. Stochastic processes and their applications, 112(2), 185200.

Rockafellar, R. T., \& Uryasev, S. (2002). Conditional value-at-risk for general loss distributions. Journal of Banking \& Finance, 26(7), 1443-1471.

Ross, S. (2015). The recovery theorem. The Journal of Finance, 70(2), 615-648.

Siegel, J. J. (1998). Stocks for the long run. New York: McGraw-Hill.

Sriboonchita, S., Wong, W.-K., Dhompongsa, S., \& Nguyen, H. T. (2009). Stochastic dominance and applications to finance, risk and economics. Boca Raton: CRC Press.

Treussard, J. (2006): The Non-Monotonicity of Value-at-Risk and the Validity of Risk Measures over Different Horizons. http://ssrn.com/abstract=776651.

Wang, S. (1996). Premium calculation by transforming the layer premium density. ASTIN Bulletin: The Journal of the IAA, 26(1), 71-92.

Wilkie, D. (2001): On the Risk of Stocks in the Long Run: A response to Zvi Bodie. In: Proceedings of the 11th International AFIR Colloquium (pp. 741-762).

Publisher's Note Springer Nature remains neutral with regard to jurisdictional claims in published maps and institutional affiliations. 